\title{
POST-OPERATIVE CARDIOVASCULAR COMPLICATIONS IN MAJOR JOINT ARTHROPLASTIES
}

Summer Syed MD, Tomas VanHelder MD PhD

Department of Anaesthesia, Hamilton Health Sciences, 1200 Main Street West, Hamilton, L8N $3 Z 5$

\section{INTRODUCTION}

Cardiovascular morbidity and mortality are associated with total joint arthroplasties given the age and burden of co-morbid illness in this population. The purpose was to evaluate and compare the frequency of clinically significant cardiovascular complications in single, staged, and bilateral hip and knee arthroplasties, and by type of anaesthetic.

\section{METHODS}

Patient's demographic data, cardiovascular co-morbidities, anaesthetic type, type of arthroplasty, and post-operative complications were identified. Statistical analysis was performed by t-test or Chisquare. Regional anaesthesia (RA) was defined as spinal, epidural or combined spinal-epidural. Failed regional technique requiring conversion to general anaesthetic was counted as a general anaesthetic (GA). Bilateral arthroplasties were done sequentially under one anaesthetic. Staged arthroplasties were done within 4 years of one another.

\section{RESULTS}

2209 charts were analysed in the periods of January 1995 - March 1996 and September 1998 January 2001. Statistical power was $>80 \%$ with $\alpha$ error 5\%. 1925 patients had primary arthroplasty; 926 were single knees, 747 single hips, 9 bilateral hips, 240 bilateral knees, 3 combined hip and knee. 284 were revision arthroplasties and are not included in this report.

Patient populations were not significantly different between hip and knee arthroplasties except for diabetes mellitus, which was higher in knee patients. Hip patients were younger (67.8) than knee patients $(69.8)(\mathrm{p}<0.001)$. No differences were found in total cardiovascular complications between single hip (7.5\%) and knee (8.6\%) procedures, and between anaesthetic types (9\% RA, 8.6\% GA). Bilateral knees had higher complications $(15.8 \%)$ than single knees $(8.6 \%)$, or staged knees $(7.0 \%)$ $(\mathrm{p}<0.001)$. Bilateral hips and knees had more complications $(16.7 \%)$ than staged procedures $(7.0 \%)$ $(\mathrm{p}<0.001)$. Complications in single $(7.5-8.6 \%)$ and staged $(7.0 \%)$ procedures were similar.

\section{DISCUSSION}

Bilateral arthroplasties result in higher cardiovascular complications compared to single or staged procedures. This is independent of the type of anaesthetic or arthroplasty. Ours is the first statistically powered study to show that bilateral arthroplasties result in higher complications than staged procedures. The exposure to two anaesthetics and two surgeries does not increase the risk of cardiovascular complications whereas the type of surgical procedure does. Thus, patients for bilateral arthroplasties should be carefully selected. 